\title{
Los ammonites del Caloviano de la región Mixteca, Oaxaca, México
}

\author{
Raúl Sabino Carrasco-Ramírez \\ Instituto Nacional de Estadística, Geografía e Informática \\ Dirección Regional Noreste \\ Pino Suárez \# 790 Sur, 64000 Monterrey, N. L., México \\ rcarrasco@sysop.com.mx
}

\section{Resumen}

En la región Mixteca, al noroeste del Estado de Oaxaca, sur de México, afloran rocas del Jurásico Medio con una fauna fósil que consiste en su mayor parte de ammonoideos. En el Miembro Yucuñuti de la Formación Yucuñuti, fueron identificadas varias facies sedimentarias compuestas principalmente por: cuarzarenita, limolita y biomicrita, correspondientes a mares neríticos someros o epicontinentales con aporte de abundantes terrígenos. Se identificó la Zona de Asociación de ReineckeiaNeuqueniceras (Nueva), que se caracteriza por la predominancia de Reineckeidae y se correlaciona con la Zona Anceps del Caloviano medio de Europa. Se describen cinco ejemplares de las familias Perisphinctidae y Reineckeidae: Ch. cf. suborion (Burckhardt), Ch. praecursor (Mangold), N. inermis (Burckhardt), R. anceps (Reinecke) y R. tuberosa (Burckhardt). Se informa por primera vez para México la presencia de la especie $R$. anceps, índice de la Zona Estándar de Anceps del Caloviano medio temprano de Europa.

Palabras clave: ammonites, Formación Yucuñuti, asociación, Caloviano, México.

\begin{abstract}
In the Mixteca region, in northwestern Oaxaca state, Mexico, crop out Middle Jurassic rocks with a rich fossil fauna, consisting mostly of ammonoids. The CallovianYucuñuti Member of the Yucuñuti Formation, consists of nearshore to shelf siltstone, sandstone grit and biomicrite. Ammonoids present in this unit belong in the Reineckeia-Neuqueniceras Assemblage Zone (nov.), which is coeval to the European Anceps Zone, Stuebeli Subzone (base of the middle Callovian). They belong to the Perisphinctidae and Reineckeidae families, and include the following species: Ch. cf. suborion (Burckhardt), Ch. Praecursor (Mangold), N. inermis (Burckhardt), R. anceps (Reinecke) and R. tuberosa (Burckhardt). Reineckeia anceps, the index species of the European Anceps Zone of the early middle Callovian, is described for the first time for Mexico.

Key words: ammonoids, Formación Yucuñuti, assemblage, Callovian, Mexico.
\end{abstract}




\section{Introducción}

En el presente trabajo se describen rocas del Caloviano, y sus ammonites, que afloran en las áreas de San Juan Mixtepec y Santa María Asunción Tlaxiaco (Figura 1). Estas localidades se ubican en la región Mixteca, al noroeste del Estado de Oaxaca, único lugar donde es posible estudiar en su conjunto las rocas del Jurásico Medio del sur de México.

La mayor parte de los afloramientos de la Mixteca corresponden a rocas metamórficas paleozoicas del Complejo Acatlán, rocas sedimentarias de la Caliza Teposcolula del Albiano-Cenomaniano, rocas volcánicas del Terciario y, en menor medida, a rocas sedimentarias del Jurásico Superior. Rocas del Caloviano están presentes en áreas relativamente restringidas, formando parte de la Formación Yucuñuti, la cual consta de tres miembros que de la base a la cima son: Miembro Simón, Miembro Otatera y Miembro Yucuñuti (Carrasco-Ramírez, 1999). El Caloviano corresponde al Miembro Yucuñuti, el cual aflora en toda su extensión en las áreas de estudio y en donde fueron colectados ammonites para su estudio taxonómico. El objetivo del presente trabajo es describir las rocas del Caloviano y sus ammonites.

Si bien en la región Mixteca se han desarrollado numerosos trabajos de investigación, tanto geológicos como paleontológicos, incluyendo estudios sobre los ammonites,
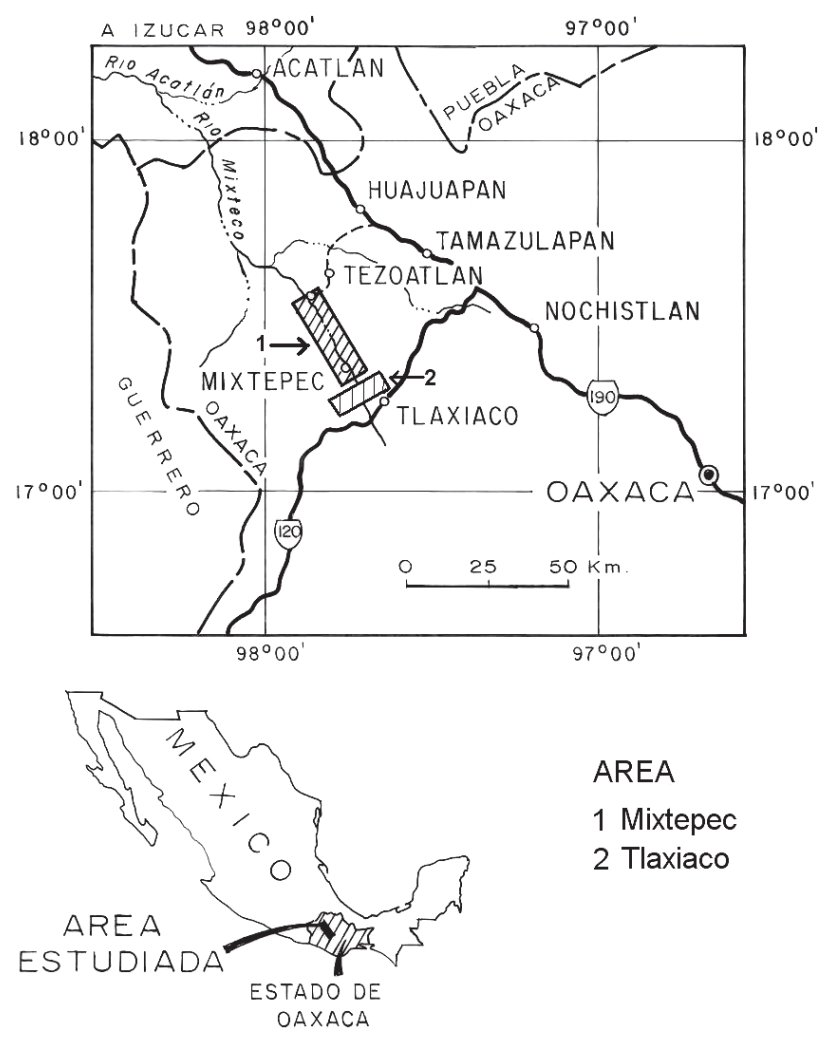

Figura 1. Mapa de localización del área de estudio, en el noroeste del Estado de Oaxaca. las secciones aquí presentadas y su fauna se estudian por primera vez.

Debido a que la región Mixteca contiene depósitos de carbón, ha sido objeto de estudios de geología económica relacionados con este material desde el siglo pasado. En dichos estudios se encuentran esbozos de la geología y de la presencia de fósiles. Felix (1891) mencionó en varias ocasiones la existencia de afloramientos cerca de Tlaxiaco, que contienen ammonites indicativos del Jurásico Medio. Esta publicación incluye el estudio de moluscos gasterópodos y bivalvos, así como de plantas fósiles. Flores (1909), en sus exploraciones geológicas en el oeste de Oaxaca y el este de Guerrero, recolectó ammonites y obtuvo datos geológicos que contribuyeron al conocimiento incipiente de la región. El material recolectado por él fue proporcionado a Carl Burckhardt, quien lo estudió junto con otros ejemplares que él mismo colectó, publicando el primer trabajo sobre ammonites de la región (Burckhardt, 1927), con el que sentó las bases de la paleontología del Jurásico Medio del sur de México. Sin embargo, aunque la fauna está excelentemente descrita e ilustrada en su monografía, los ammonites carecen de control estratigráfico preciso.

Mucho tiempo después Erben (1956) emprendió el estudio geológico del noroeste de Oaxaca, estableciendo la nomenclatura litoestratigráfica de la región. Sin embargo, algunas de las unidades formacionales que describió no se reconocen en campo, dado su carácter transicional marinocontinental, correspondiendo en realidad a diferentes facies de la misma formación. También recolectó conjuntos faunísticos similares a los descritos 30 años antes por Burckhardt (1927), pero no ilustró ni describió los fósiles, $\mathrm{y}$ tampoco indicó su ubicación litoestratigràfica.

\subsection{Litoestratigrafía de las secciones estudiadas}

Carrasco-Ramírez (1981), al estudiar el área de Tlaxiaco, trabajó en el Miembro Yucuñuti de la Formación Yucuñuti, que aflora con un espesor de $118 \mathrm{~m}$ y consiste de arenisca, coquina negra y caliza en capas delgadas con bivalvos y gasterópodos. En esa investigación, al efectuar el estudio petrográfico de la secuencia fueron identificadas principalmente las siguientes litofacies :

1) Cuarzarenita (lutita amarilla-arenisca verde), depositadas probablemente en una llanura de inundación, luego de un transporte relativamente prolongado desde un área de aporte constituida por rocas ígneas y metamórficas.

2) Limolita con coquinas de invertebrados, depositada muy posiblemente en un ambiente lagunar conectado al mar.

3) Biomicrita, depositadas probablemente en mares neríticos someros o epicontinentales, que tuvieron aporte de terrígenos.

En la investigación realizada en el área de Mixtepec, cercana a la mencionada anteriormente, Carrasco-Ramírez (1999) encontró que el Miembro Yucuñuti aflora en la 
localidad de El Llano, municipio de San Juan Mixtepec (Figura 2). En este lugar se estudiaron las localidades El Llano 1 y El Llano 2.

\subsubsection{El Llano 1}

Se ubica en el flanco norte del Cerro El Llano, aflora a $2.5 \mathrm{~km}$ al noroeste de El Llano, municipio de San Juan Mixtepec. La sección con ammonites se describe a continuación de la base a la cima, con niveles en paréntesis (Figura 3).

(Lutita amarilla o café sin ammonites de la Formación Taberna)

0) $15 \mathrm{~m}$. Lutita amarilla conteniendo especímenes enteros de los bivalvos C. hayamii Alencaster y Eocallista imlayi Alencaster.

1) $10 \mathrm{~m}$. Arenisca verde claro que intemperiza a rojo, sin macrofósiles.

2) $6 \mathrm{~m}$. Arenisca algo calcárea y arcillosa de color amarillo con pocos ammonites. Se colectó un fragmento de $R$. tuberosa y otro de $R$. cf. grossouvrei.

3) $10 \mathrm{~m}$. Lutita calcárea amarilla sin ammonoideos

4) $20 \mathrm{~m}$. Lutita calcárea amarilla con algunos ammonites. Se colectó en la cima de este nivel in-situ y entero el espécimen $C$. inermis y en la parte media dos ejemplares de $R$. tuberosa.

Pocos metros arriba sigue en discordancia la Caliza con "Cidaris".

\subsubsection{El Llano 2}

Se ubica en el flanco oeste del cerro El Llano, aflora a $1.5 \mathrm{~km}$ de El Llano, municipio de San Juan Mixtepec. A continuación se describe la sección con ammonites de la base a la cima, con niveles en paréntesis (Figura 4).

(Arenisca y lutita sin ammonites de la parte basal del Miembro Yucuñuti)

1) $5 \mathrm{~m}$. Arenisca calcárea sin macrofósiles.

2) $5 \mathrm{~m}$. Arenisca y limolita calcárea con coquina y los ammonites $C$. praecursor y $R$.(R.) anceps.

3) $7 \mathrm{~m}$. Arenisca calcárea en estratos de 10-20 cm; se colectó un espécimen completo de $N$. (F.) cf. bodenbenderi.

4) $5 \mathrm{~m}$. Arenisca y limolita calcárea con coquina y ammonites. Un espécimen completo de $R$. (R.) anceps y un fragmento ex-situ de $N$. cf. neogaeum.

5) $4 \mathrm{~m}$. Limolita calcárea en estratos delgados de 20 $50 \mathrm{~cm}$, un fragmento ex-situ del espécimen "P." cricotum.

6) $10 \mathrm{~m}$. Limolita que intemperiza en estratos suaves y limolita calcárea en capas delgadas de 5 a $10 \mathrm{~cm}$., con $R$. (R.) anceps,, el bivalvo Trigonia (Indotrigonia) impressa (Sowerby), R. tuberosa, C. cf. suborion y N. (F.) cf. bodenbenderi.

7) $10 \mathrm{~m}$. Arenisca con coquina, limolita arenosa y limolita calcárea en capas delgadas de $10 \mathrm{a} 20 \mathrm{~cm}$ con un espécimen ex-situ entero de C. praecursor. Siguen pocos metros arriba en discordancia afloramientos de la Caliza con "Cidaris".

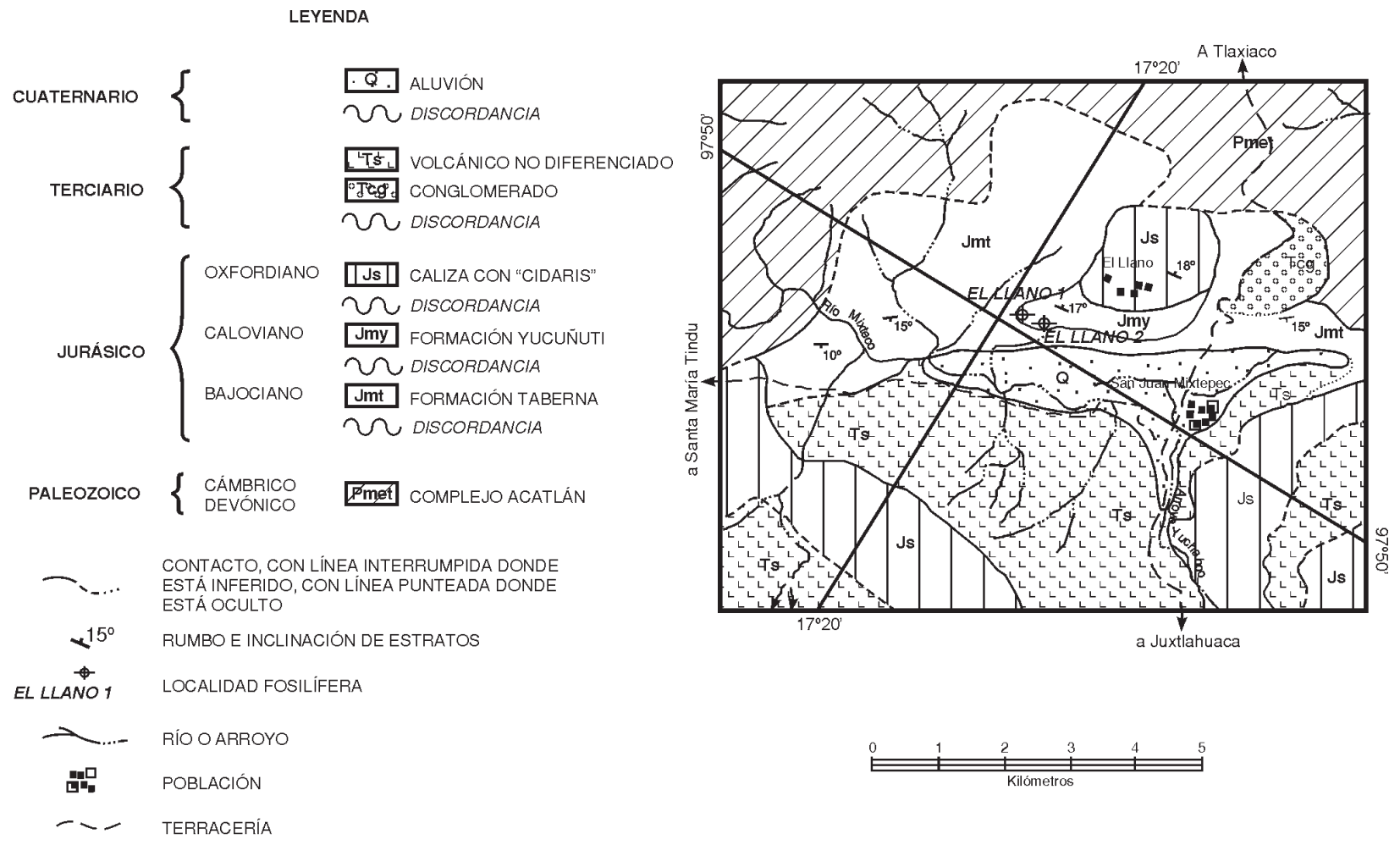

Figura 2. Mapa geológico del área de Mixtepec. Muestra las localidades del Caloviano El Llano 1 y El Llano 2. Tomado de Carrasco-Ramírez (1999). Base topográfica tomada de la hoja E14D34 editada por el INEGI, geología realizada por Raúl S. Carrasco-Ramírez. 


\section{Paleontología sistemática}

Todos los ejemplares de ammonites que se encontraron en las secciones muestreadas, fueron sujetas a un estudio taxonómico. Sin embargo, la descripción de la mayor parte de ellas no se incluye en este trabajo, puesto que se encuentran bien descritas en otros trabajos a los que se hace referencia en el texto (Westermann et al., 1984; Sandoval et al., 1990). Asimismo, se consideró conveniente proporcionar ilustraciones de las especies que no fueron figuradas en los mencionados trabajos.

Por lo anteriormente señalado, este capítulo comprende únicamente la descripción de cinco especies que se seleccionaron por diversas razones. Así, se describe una especie que fue encontrada por primera vez en México $[R$. (R.) ancep]. Igualmente, $R$. tuberosa, porque su sinonimia se modifica, al considerarse como sinónimos once nombres específicos. La descripción de las otras especies obedece, o bien a un cambio genérico ( $N$. inermis), o a que se modifican ligeramente o se completan las descripciones anteriores, por contarse con material mejor conservado. Todas las especies descritas proceden del Caloviano de la región Mixteca de Oaxaca, pertenecen a la superfamilia Perisphinctacea, y están incluidas en dos familias y en tres géneros. Todo el material se depositó en la Colección Paleontológica del Instituto de Geología de la UNAM (IGM).

Las dimensiones están en milímetros, y se utilizaron las abreviaturas convencionales, excepto $\mathrm{P}$ (número de costillas primarias por media vuelta) y $\mathrm{S}$ (número de costillas secundarias por media vuelta).

Superfamilia Perisphinctoidea Steinmann, 1890. Familia Perisphinctidae Steinmann,1890. Subfamilia Grossouvriinae Spath, 1930 Género Choffatia Siemiradzki, 1898.

Especie tipo. Perisphinctes cobra, Waagen, 1869, por designación original, p.171, lám. 45, figs. 1, 1a.

\section{Choffatia cf. suborion (Burckhardt)} Figuras 5 y 6 (A, B)

cf. 1927 Perisphinctes suborion Burckhardt, p. 77, lám. 31, figs. 2-4, 7.

cf. 1990 Choffatia suborion (Burckhardt, 1927). Sandoval et al., p. 130, lám. 10, fig. 2, lám.12, figs. 1, 2 a-b.

Descripción. Fragmento de fragmocono que comprende menos de la cuarta parte de la concha; que contiene vuelta externa y porciones de dos vueltas internas. Corresponde a una concha evoluta, con vueltas que abrazan una tercera parte de los flancos de la vuelta anterior, con ombligo amplio y poco profundo, de flancos aplanados y borde umbilical angular redondeado, de paredes suavemente inclinadas y

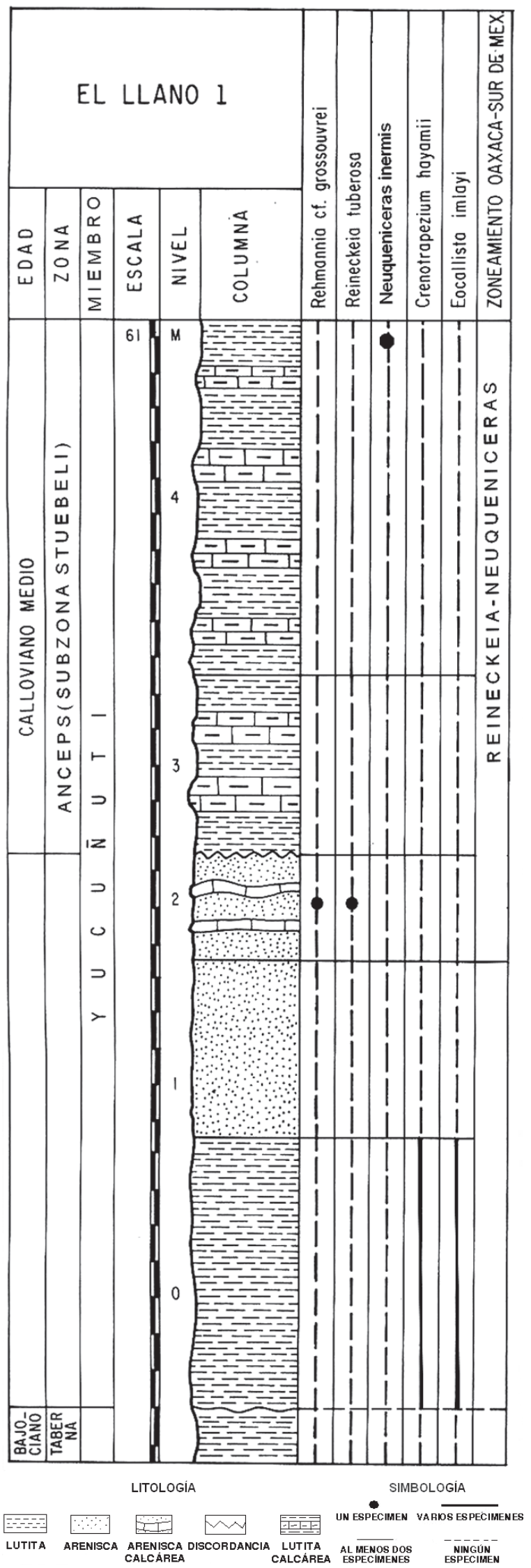

Figura 3. Columna estratigráfica del norte del Cerro El Llano, en la localidad de El Llano 1. 


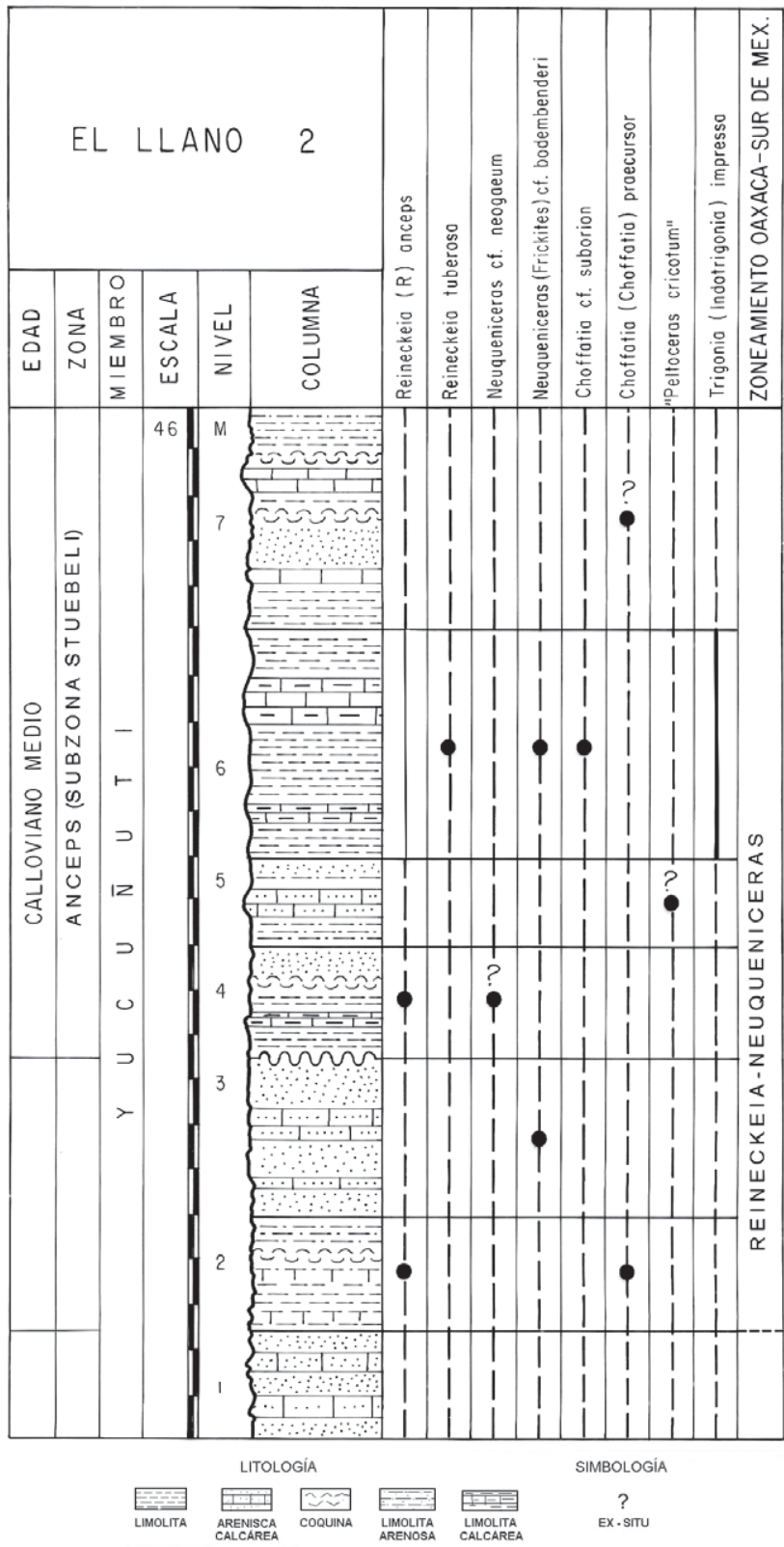

Figura 4. Columna estratigráfica del oeste del Cerro El Llano, en la localidad de El Llano 2.

bajas en las vueltas internas y alto convexo en la última vuelta. La sección de las vueltas es subcuadrangular, más alta que ancha la última vuelta y tan ancha como alta la penúltima conservada. Región ventral moderadamente convexa.

La ornamentación en las vueltas internas muestra costillas primarias radiales, simples, prominentes, redondeadas, separadas por espacios intercostales ligeramente más anchos que las costillas. En la última vuelta las costillas primarias son prominentes, como crestas angulares radiales, sin tubérculos, que se extienden desde el reborde umbilical hasta la mitad del flanco, donde se

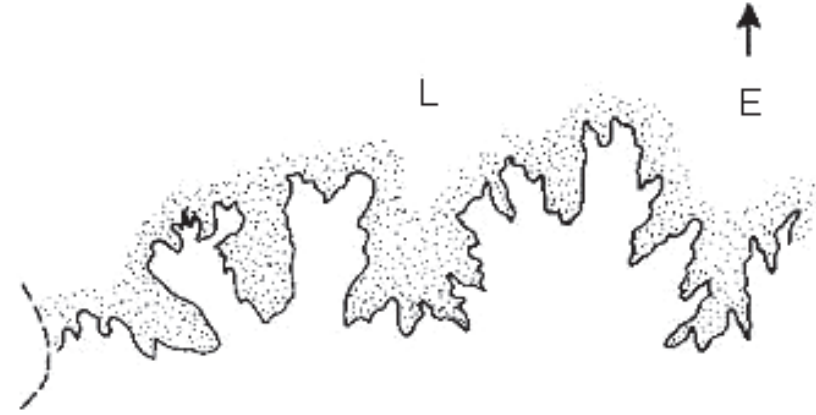

Figura 5. Línea de sutura de Choffatia cf. suborion (Burckhardt). Clave IGM 7771 a $\mathrm{D}=150 \mathrm{~mm}$ aprox.

dividen en dos o tres secundarias, o bien, en dos más una intercalada, resultando tres secundarias por cada primaria; están inclinadas ligeramente adaperturalmente. En el centro de la región ventral se interrumpen en una banda lisa. Solamente el flanco derecho presenta dos constricciones adaperturales, limitadas adelante por una costilla principal $\mathrm{y}$ atrás por dos costillas secundarias rectas.

La sutura (Figura 5) consiste de un lóbulo externo (E) poco profundo e indentado, lóbulo lateral (L) con igual profundidad que $\mathrm{E}$ y poco indentado, primer lóbulo auxiliar $\left(U_{2}\right)$ igual de profundo a $(L)$, pero angosto, y segundo lóbulo auxiliar $\left(\mathrm{U}_{3}\right)$ retraido.

Discusión. El espécimen que se describe consiste de un fragmento, la cuarta parte de una concha. Por tal motivo nada más se establece la comparación, dadas las características similares de este fragmento con la especie descrita por Sandoval et al.(1990). Estas similitudes son principalmente las costillas primarias radiales, prominentes como crestas que se extienden, en la última vuelta, desde el borde umbilical hasta la mitad del flanco dividiéndose en dos o tres secundarias, también presentan en la mitad del vientre una banda lisa.

Material y ocurrencia. El fragmento que se describe proviene de la parte superior de la localidad El Llano 2. El alcance estratigráfico fue asignado por Sandoval et al. (1990) en el Batoniano superior, pero dada la nueva asociación en la que se encuentra, en este trabajo se asigna al Caloviano medio.

\section{Choffatia (Choffatia) praecursor Mangold 1970} Figuras 6 (C, D, E), 7 y 8 ( A, B)

1958 Siemiradzkia (Siemiradzkia) subfurcula (Liss.), Westermann, p. 81, lám. 40, fig.1a.

1958 Choffatia (Choffatia) sp. b, Westermann, p. 84, lám. 42 , fig. 1.

1970 Choffatia (Choffatia) praecursor Mangold, p. 139, fig. 96, 97, lám.2, fig.4. 

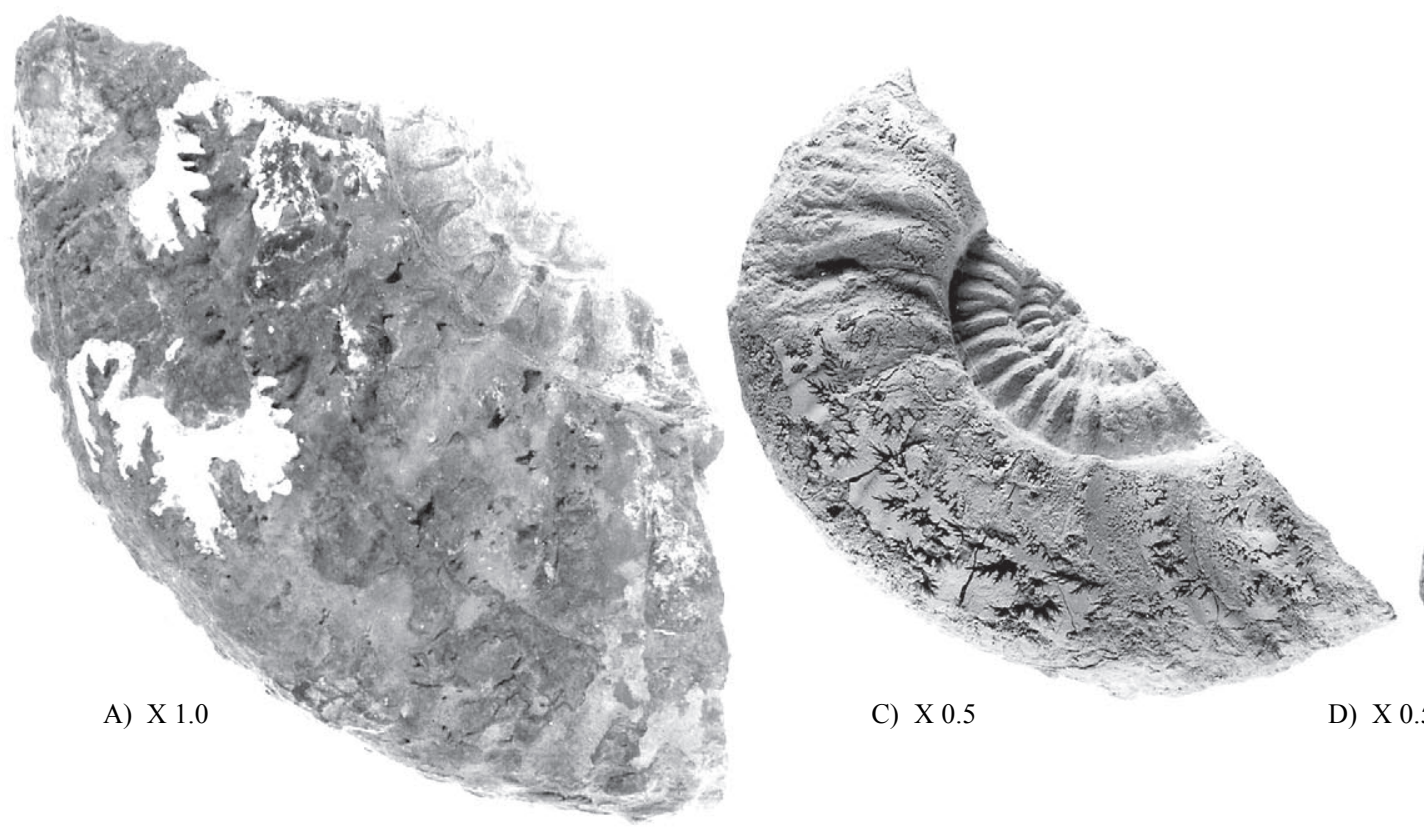

C) $\mathrm{X} 0.5$

D) $\mathrm{X} 0.5$
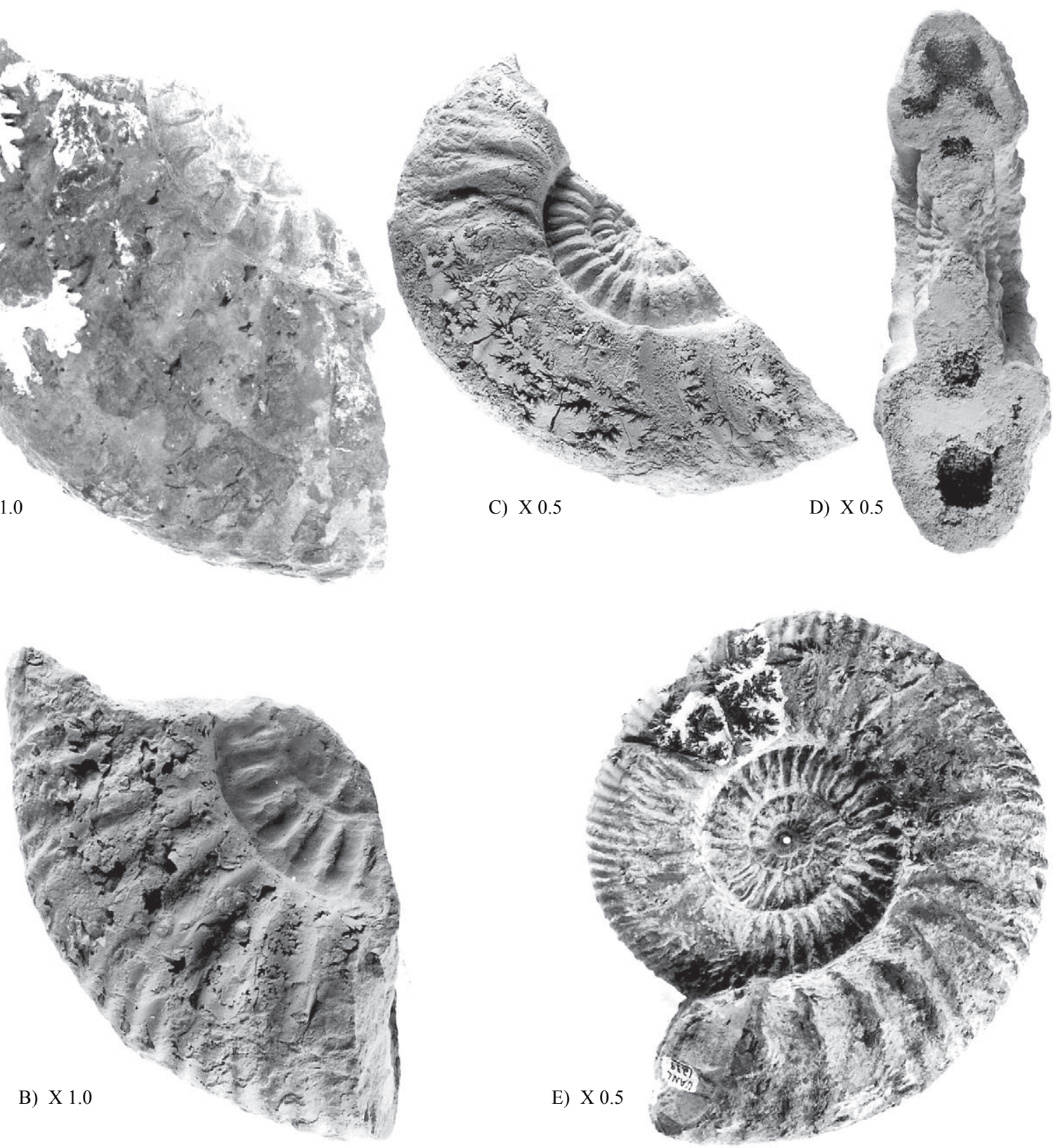

E) $\mathrm{X} 0.5$

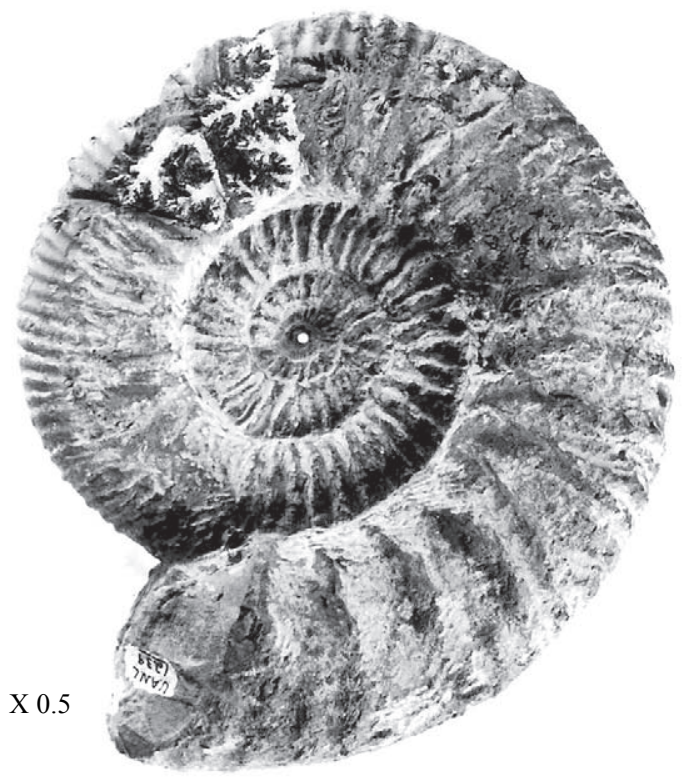

Figura 6. Choffatia cf. suborion Burckhardt, IGM-7771, localidad El Llano 2, de la parte media del Miembro Yucuñuti; A) vista latero-ventral que muestra detalle de la sutura (X 1); B) Vista lateral (X 1). Choffatia (Choffatia) praecursor Mangold, IGM-7772, localidad El Llano 2, en la parte media de la Formación Yucuñuti; C) vista lateral (X 0.5); D) vista en sección (X 0.5); E) IGM-7773, vista lateral que muestra detalle de la sutura (X 0.5).

Descripción. Macroconcha, evoluta; la vuelta externa abraza un poco menos de la mitad de la vuelta anterior. El ombligo es amplio y profundo, con el muro umbilical alto y vertical y el reborde umbilical redondeado. La última vuelta se expande rápidamente. La sección de las vueltas internas es oval subcircular, con los flancos poco convexos y la región ventral convexa. En cambio, la sección de la última vuelta, de flancos planos, convergentes hacia el vientre, que es convexo y mucho más estrecho que la base de la sección.

La ornamentación en las vueltas internas presenta costillas primarias radiales, prominentes y elevadas, redondeadas, (quince en la mitad de la penúltima vuelta de un ejemplar de $190 \mathrm{~mm}$ de diámetro), separadas por espacios intercostales cóncavos, del mismo ancho que las costillas. En la mitad de la última vuelta, las costillas primarias son muy prominentes, en forma de crestas redondeadas sin tubérculos, extendidas desde la línea umbilical hasta el primer tercio de los flancos, donde se dividen en dos o tres costillas secundarias poco salientes, que son desvanecidas hacia la región ventral y se interrumpen en el centro en un 


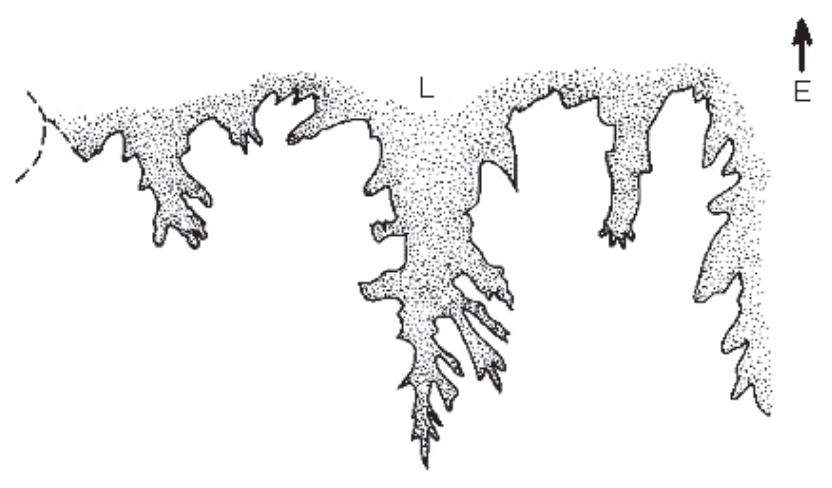

Figura 7. Línea de sutura de Choffatia praecursor (Manngold). Clave IGM 7772 a $\mathrm{D}=176 \mathrm{~mm}$.

espacio liso angosto. Las costillas primarias en la última vuelta son curvas, con un arqueamiento convexo hacia atrás de la abertura, menos numerosas y con espacios intercostales más amplios; las secundarias son rectas, ligeramente inclinadas adaperturalmente.

\begin{tabular}{lccccccccc}
\hline \multicolumn{10}{c}{$\begin{array}{c}\text { Dimensiones } \\
(\mathrm{mm})\end{array}$} \\
\hline Ejemplar & $\mathrm{D}$ & $\mathrm{U}$ & $\mathrm{H}$ & $\mathrm{W}$ & $\mathrm{U} / \mathrm{D}$ & $\mathrm{H} / \mathrm{D}$ & $\mathrm{W} / \mathrm{D}$ & $\mathrm{P}$ & $\mathrm{S}$ \\
\hline IGM-7772 & 190 & 76 & 67 & 58 & 0.40 & 0.35 & 0.31 & 12 & - \\
IGM-7773 & 151 & 63 & 47 & 48 & 0.42 & 0.31 & 0.32 & 12 & 33 \\
\hline
\end{tabular}

La sutura (Figura 7) consiste en lóbulos estrechos, indentados, simétricos y profundos, con características típicas del género Choffatia.

Discusión. La descripción anterior está basada en dos fragmoconos, y se carece de la cámara de habitación. $C$. praecursor es muy semejante a $C$. vicenti Mangold. Ambas especies se encuentran en el mismo nivel estratigráfico del Batoniano superior en Europa y se diferencian en que la primera presenta una ornamentación más prominente de costillas primarias que no son radiales, sino arqueadas hacia atrás en el reborde umbilical e inclinadas adaperturalmente en los flancos y en la región ventral.

También es semejante a C. burckhardti (Sandoval et al., 1990, p. 129, lám. 11, fig, 1 a, b) del Batoniano superior de la Formación Yucuñuti de Cualac, Guerrero, en las dimensiones y en el perfil de las vueltas. Ambas especies diferenciadas porque en $C$. burckhardti las costillas son radiales y las primarias más espaciadas en las vueltas internas.

C. praecursor también es comparable con $C$. suborion (Burckhardt, 1927, p. 77, lám. 31, figs. 2-4, 7; Sandoval et al., 1990, p. 130, lám. 10, fig. 2, lám. 12 figs. 1 y 2) y con C. jupiter (Steinmann) (Riccardi et al., 1989, lám. 5, fig. 1) presentes en el Caloviano de Mixtepec, Oaxaca, de Cualac, Guerrero y de los Andes Argentino-Chilenos, respectivamente. Pero estas especies no son masivas, la vuelta externa no es muy expandida, siendo más de dos veces mayor que la vuelta anterior, con ornamentación radial y el perfil de las vueltas oval-circular.

Mangold (1970, p. 136) indica que el género Choffatia aparece en la base del Batoniano superior y se extiende hasta la parte superior del Caloviano medio.

Material y Ocurrencia. Medio espécimen medianamente preservado (IGM 7772) y otro (IGM 7773) completo y muy bien preservado. Ambos provienen de los niveles inferior y superior del Miembro Yucuñuti, en la localidad El Llano 2. $\mathrm{Su}$ alcance estratigráfico se indica en el párrafo anterior (Mangold, 1970), no obstante la nueva asociación que se encontró en este trabajo, la posición que le corresponde a esta especie es del Caloviano medio.

Familia Reineckeiidae Hyatt, 1900

Subfamilia Neuqueniceratinae Cariou, 1984

Género Neuqueniceras Sthen, 1923

Especie tipo. Neuqueniceras steinmanni Stehn, 1923

Neuqueniceras inermis (Burckhardt, 1927) Figura $8(\mathrm{C}, \mathrm{D})$

1927 Reineckeia inermis Burckhardt, p.70, lám. 28, figs. $1,2,7$.

Descripción. Concha de tamaño medio, evoluta, discoidal, moderadamente aplanada; las vueltas abrazan aproximadamente la mitad de la vuelta anterior. El ombligo es amplio, moderadamente profundo; la sección de las vueltas es subrectangular ovalada, la parte umbilical es elevada, vertical, con reborde umbilical redondeado, los flancos aplanados y la región ventral algo convexa.

La ornamentación es muy regular. Consiste en costillas primarias prominentes, redondeadas a ligeramente agudas, que se extienden desde el borde umbilical hasta el centro de la región ventral, donde se interrumpen en una angosta banda lisa. Son casi rectas, ligeramente prorsiradiadas, con una ligera curvatura cóncava a la abertura desde el borde umbilical hasta el primer tercio del flanco. Carecen de tubérculos; están espaciadas regularmente, separadas por espacios intercostales lisos, del doble del ancho de las costillas.

En la vuelta externa, las costillas primarias se dividen aproximadamente en la parte media de los flancos en dos o tres secundarias casi rectas, con una ligera inclinación adapertural, igual que las primarias, e igualmente se interrumpen en el centro en una angosta banda lisa.

En la última vuelta hay cuatro constricciones profundas, más anchas que los interespacios, limitadas adelante (adaperturalmente) por una costilla prominente simple y atrás por costillas secundarias bifurcadas. 


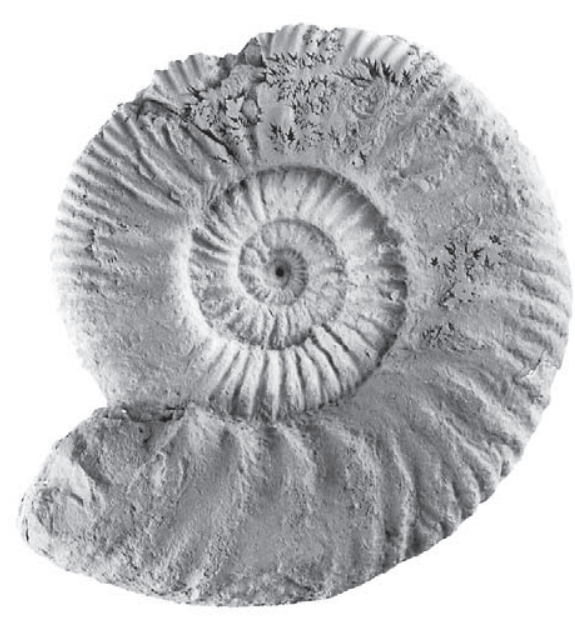

A) $\mathrm{X} 0.50$
B) $\mathrm{X} 0.50$
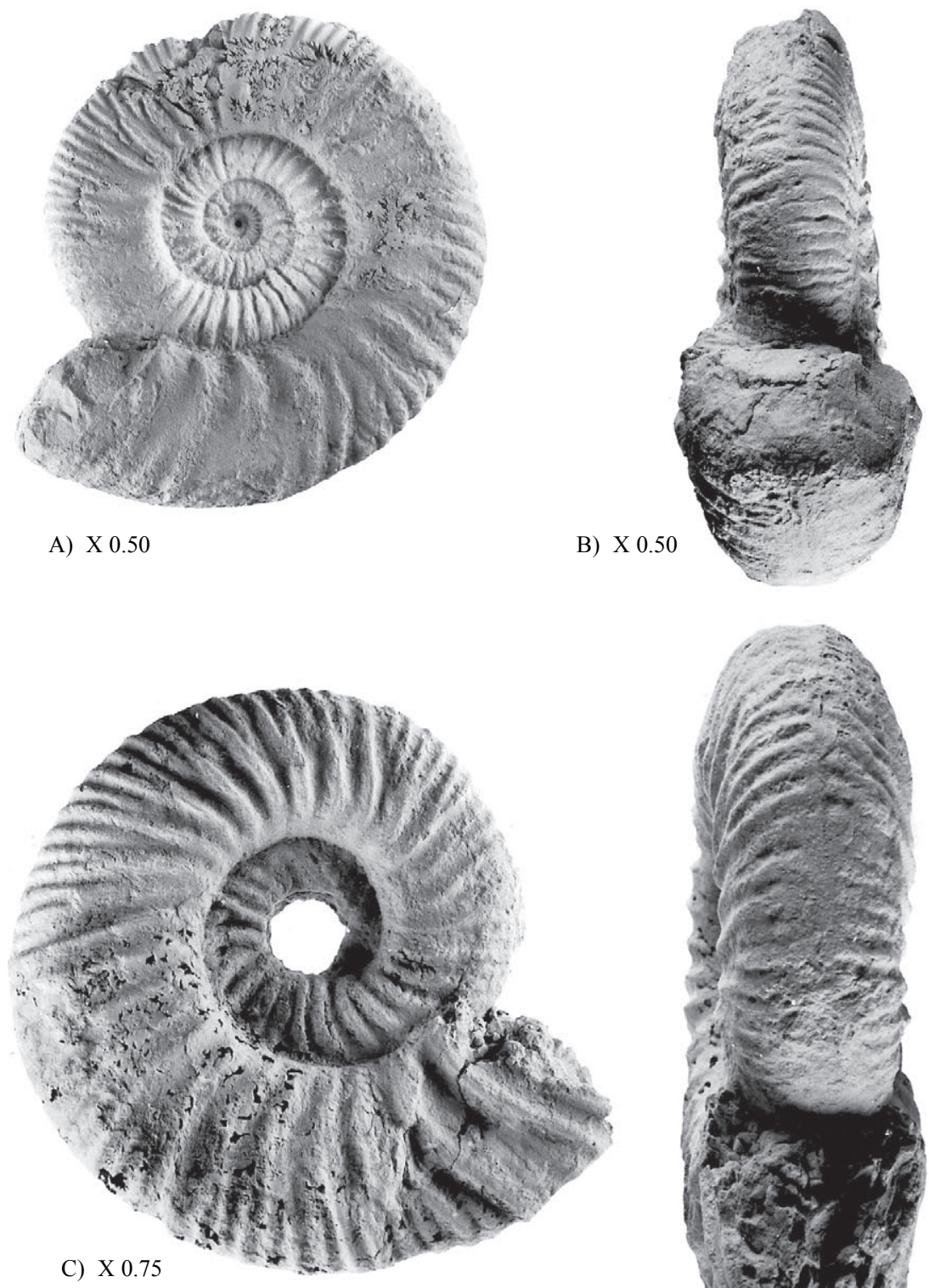

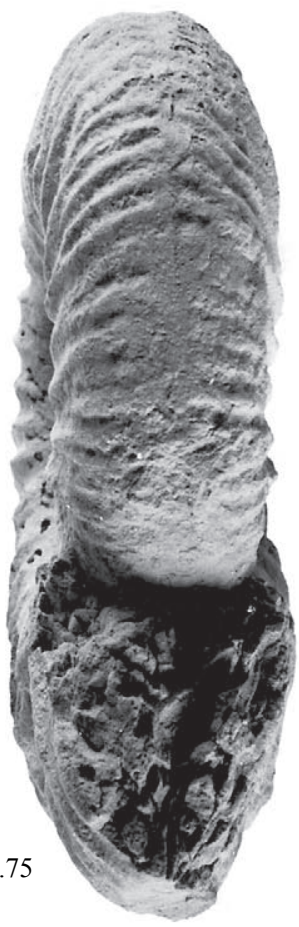

Figura 8. Choffatia (Choffatia) praecursor Mangold, IGM-7773, localidad El Llano 2, en la parte media del Miembro Yucuñuti. A) Vista lateral (X 0.5); B) Vista por la abertura (X 0.5). Neuqueniceras inermis Burckhardt, IGM-7770, localidad El Llano 1, de la parte media de la Formación Yucuñuti; C) vista lateral (X 0.75); D) vista por la abertura (X 0.75).

\begin{tabular}{lccccccccc}
\hline \multicolumn{10}{c}{$\begin{array}{c}\text { Dimensiones } \\
(\mathrm{mm})\end{array}$} \\
\hline Ejemplar & D & U & H & W & U/D & H/D & W/D & P & S \\
IGM-7770 & 99 & 38 & 33 & 32 & 0.38 & 0.33 & 0.32 & 14 & 36 \\
\hline
\end{tabular}

Discusión. Esta forma presenta una clara interrupción ventral de las costillas y pertenece a un Neuqueniceras.
Inicialmente fue ubicada por Burckhardt (1927) como una Reineckeia, pero con los resultados de esta investigación se le asigna en posición más adecuada.

Material y ocurrencia. Un espécimen muy bien preservado (IGM 7770), consistente en un fragmocono, aparentemente sin cámara de habitación, de la parte superior de la localidad El Llano 1. Por la relación que tiene con las demás especies que se colectaron se ubica en el Caloviano medio. 
Subfamilia Reineckeiinae Hyatt, 1900 Género Reineckeia Bayle, 1878

Especie tipo. Nautilus anceps Reinecke, 1818

\section{Reineckeia (Reineckeia) anceps (Reinecke)}

Figuras 9 y 10 ( A, B, C, D)

1980 Reineckeia (Reineckeia) anceps (Reinecke 1818). Cariou, p. 375, lám. 33, figs. 4, 5 láms. 34 - 36; lám. 37 , figs. 1, 2, por designación original, (con sinonimia).

1988 Reineckeia (Reineckeia) anceps (Reinecke 1818). Cariou y Krishna, p.160, lám. 2, fig. 2 a-b, 3; lám.3, fig. 1 a-b.

Descripción. Concha de tamaño grande, evoluta; la vuelta externa abraza aproximadamente una tercera parte de la vuelta anterior. Ombligo amplio y profundo. La última vuelta mucho más alta y ancha que la anterior. La sección de las vueltas internas es subcircular a oval $(\mathrm{D}=55 \mathrm{~mm})$. En un ejemplar grande $(\mathrm{D}=135 \mathrm{~mm})$, la sección de la vuelta externa es reniforme, mucho más ancha que alta, con los flancos muy convexos y la región ventral poco curva, moderadamente aplanada.

La ornamentación en las vueltas interiores consiste en costillas primarias cortas, radiales, prominentes y agudas, algunas con un tubérculo pequeño en la parte media y algunas bifurcadas muy cerca de la línea umbilical. En la vuelta externa las costillas primarias son curvas hacia atrás en el hombro umbilical, y después son rectas y prorsiradiadas; se dividen en el primer tercio del flanco en dos, tres o cuatro secundarias, habiendo además costillas intercaladas. Las costillas secundarias se interrumpen en el centro de la región ventral en un espacio angosto liso. Una hilera de tubérculos grandes, agudos, muy distantes, se presenta en el primer tercio de los flancos de la última vuelta, sobre la división de algunas costillas primarias, quedando

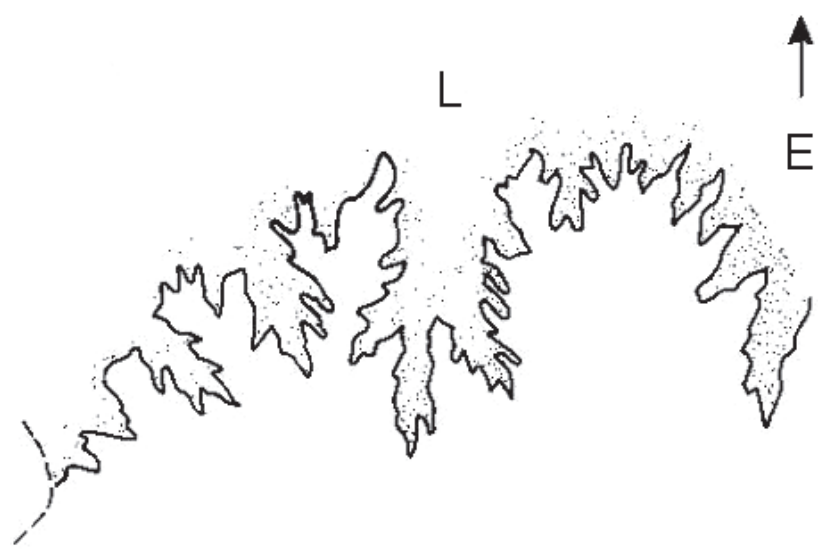

Figura 9. Línea de sutura de Reineckeia (Reineckeia) anceps (Reinecke). Clave IGM 7778 a D=90 mm. dos o tres costillas sin tubérculo. Hay constricciones profundas, inclinadas hacia adelante, muy separadas.

\begin{tabular}{lccccccccc}
\hline \multicolumn{10}{c}{$\begin{array}{c}\text { Dimensiones } \\
(\mathrm{mm})\end{array}$} \\
\hline Ejemplar & $\mathrm{D}$ & $\mathrm{U}$ & $\mathrm{H}$ & $\mathrm{W}$ & $\mathrm{U} / \mathrm{D}$ & $\mathrm{H} / \mathrm{D}$ & W/D & $\mathrm{P}$ & $\mathrm{S}$ \\
IGM-7778 & 90 & 30 & 15 & 15 & 0.33 & 0.17 & 0.17 & 10 & 52 \\
IGM-7779 & 140 & 64 & 39 & 48 & 0.46 & 0.24 & 0.34 & 6 & - \\
\hline
\end{tabular}

La sutura (Figura 9) es profundamente indentada y tiene un gran parecido a la de los representantes europeos de la especie figurados por Bourquin (1968).

Discusión. Es la primera ocasión que se registra en México la especie $R$. (R.) anceps. Se reconoce como Índice de Zona para la base del Caloviano medio del Tethys y tiene una distribución geográfica muy amplia. Se encuentra en ambos márgenes del Tethys.

Material y ocurrencia. Dos especímenes, uno casi completo (deformado) y otro que corresponde a poco más de un tercio de un individuo (IGM 7778 y 7779), provenientes del Miembro Yucuñuti, El Llano 2.

\section{Reineckeia tuberosa Burckhardt, 1927}

Figuras 11 y12 (A, B y C).

1927 Reineckeia tuberosa Burckhardt, p. 43, lám. 17, figs.1, $2,6$.

1927 R. latesellata Burckhardt, p. 45, lám. 17, figs. 3-5; lám.18, fig.1.

1927 R. coronoides Burckhardt, p. 47, lám. 18, figs. 2, 4 6.

1927 R. consolationis Burckhardt, p. 48, lám. 18, figs. 3, 7 , 8.

1927 R. sp. ind. aff. consolations, Burckhardt, p. 49, lám. 19 , figs. $1,2$.

1927 R. sp. ind. núm. 1, Burckhardt, p. 50, lám. 19, figs. 611 ; lám. 20, figs. 9,10 .

1927 R. stephanoceroides Burckhardt, p. 55, lám. 22, figs. 5-8.

1927 R. sparsinodosa Burckhardt, p. 56, lám. 23, figs. 1$3,6$.

1927 R. intercostata, Burckhardt, p.58, lám. 23, figs. 5, 79.

1927 R. wielandi Burckhardt, p. 59, lám. 23, fig. 4; lám. 24, fig. 5.

1927 R. evoluta Burckhardt, p. 69, lám. 24, figs. 1-4.

1927 R. aguilerae Burckhardt, p. 61, lám 25, figs. 1, 2.

1984 R. latesellata Burckhardt, Westermann et al., p. 108.

Descripción. Fragmento pequeño que comprende aproximadamente una quinta parte de una concha, con un pequeño fragmento de vuelta externa y de penúltima vuelta. Corresponde a una concha de tamaño medio $(\mathrm{D}=110 \mathrm{~m}$ aprox.) muy evoluta, la vuelta externa sólo abraza el vientre 

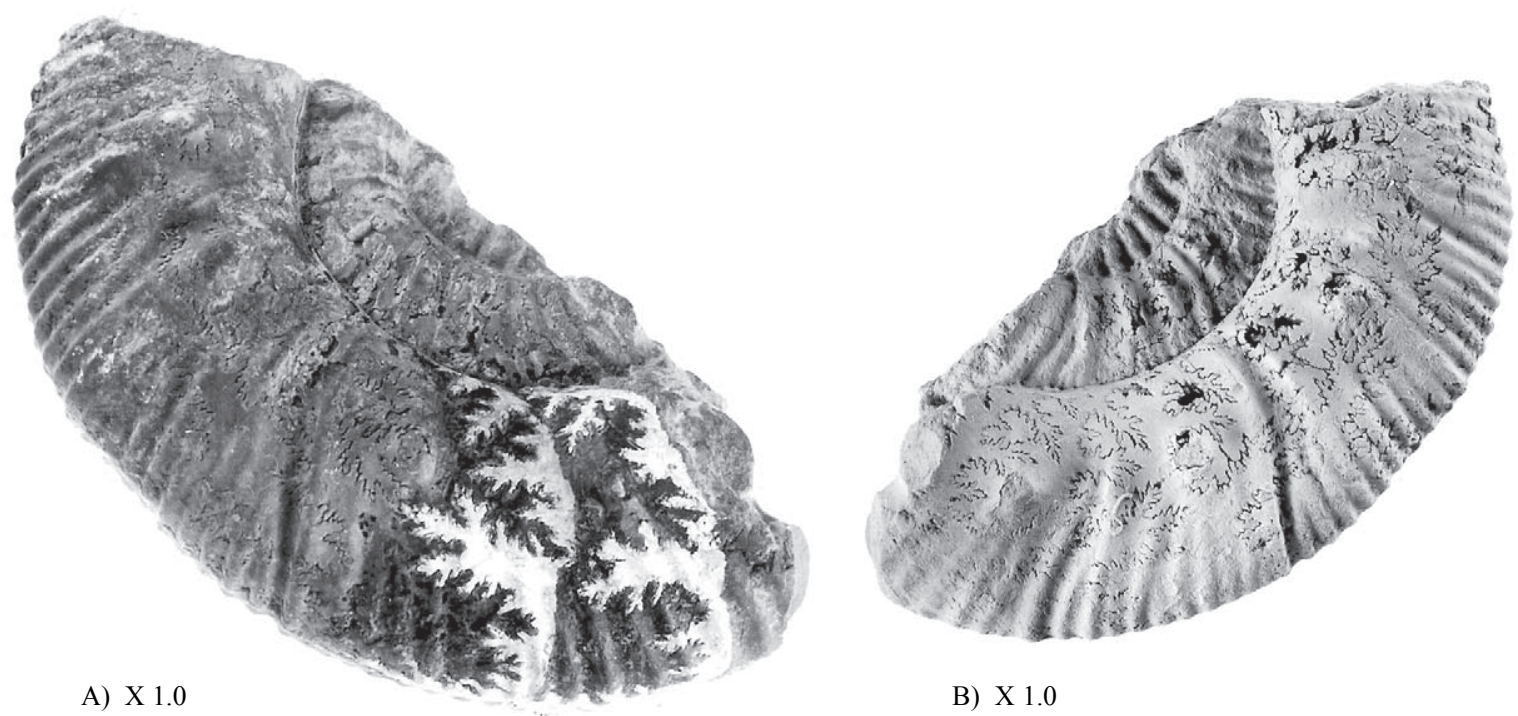

B) $\mathrm{X} 1.0$
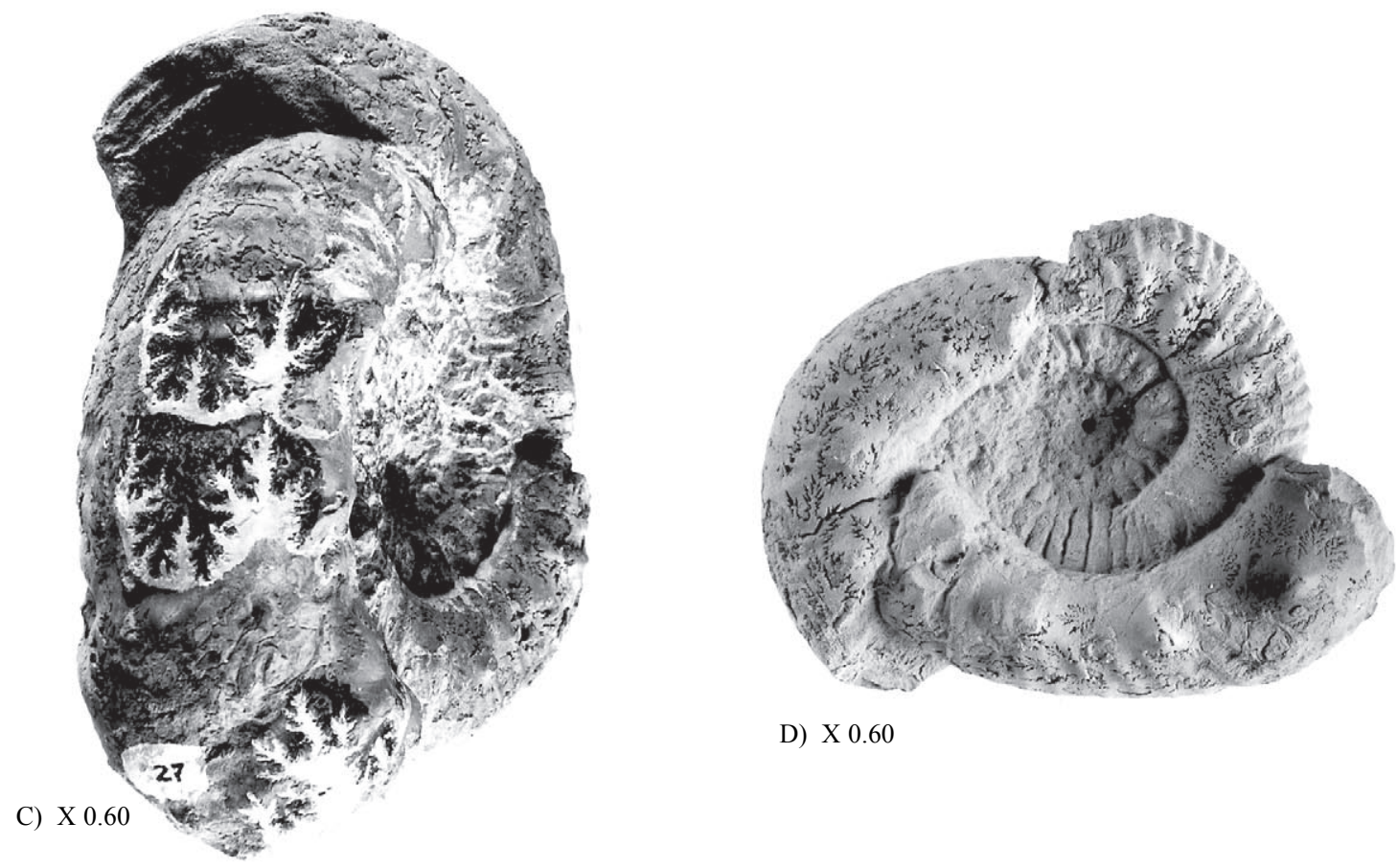

D) $\mathrm{X} 0.60$

Figura 10. Reineckeia (Reineckeia) anceps Reinecke, localidad El Llano 2, en la parte media superior del Miembro Yucuñuti; A) IGM-7778, vista lateral (X 1.0), muestra detalle de la sutura; B) vista lateral (X 1.0); C) IGM-7779, vista latero-ventral (X 0.6), muestra detalle de la sutura; D) vista lateral (X $0.6)$.

de la vuelta anterior. Ombligo amplio, poco profundo, con el declive del reborde umbilical muy tendido y poco convexo. La sección de la vuelta externa es reniforme, más ancha que alta, con los flancos convexos salientes y la región ventral aplanada; la sección de la penúltima vuelta es ovalsubcircular, tan alta como ancha, con los flancos poco salientes.

La ornamentación consiste en costillas primarias muy desvanecidas, anchas y poco elevadas, que terminan en un tubérculo prominente, aproximadamente en el tercio inferior del flanco. Los tubérculos son prominentes, con base amplia redondeada y terminados en punta. Están separados por espacios muy amplios. De cada tubérculo se originan cinco a seis costillas secundarias, cuyos extremos proximales claramente son convergentes; son redondeadas con una ligera inclinación adapertural hacia la región ventral, están distribuidas regularmente, con interespacios lisos, algo más amplios que las costillas. 


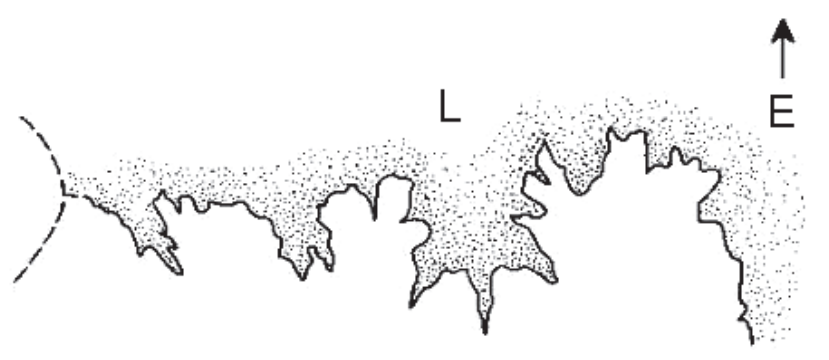

Figura 11. Línea de sutura de Reineckeia tuberosa (Burckhardt) Clave IGM 7780 a $\mathrm{D}=110 \mathrm{~mm}$ aprox.

La sutura (Figura 11) es muy semejante a la ilustrada para esta especie por Burckhardt (1927). Es indentada con el lóbulo externo (E) de la misma profundidad del lóbulo lateral (L) que es trífido y finamente denticulado; primer lóbulo auxiliar $\left(\mathrm{U}_{2}\right)$ pequeño y segundo lóbulo $\left(\mathrm{U}_{3}\right)$ auxiliar oblicuo o retraído e incisivo.

Discusión. La revisión de la colección de Burckhardt (1927) en la colección paleontológica del Instituto de Geología, permitió llegar a la conclusión de que todos los nombres en la lista de la sinonimia corresponden a una sola especie de Reineckeia. Esta colección comprende ejemplares muy bien conservados, muchos de ellos completos, con muy pequeñas diferencias que se pueden considerar como variaciones intraespecíficas. Al nombre $R$. tuberosa le corresponde la prioridad de página. Westermann et al. (1984, p. 108) consideran que aproximadamente veinte nombres de "especies" de Reineckeia de Burckhardt (1927) probablemente pertenecen a una única especie, para la que seleccionaron el nombre $R$. latesellata.

Material y ocurrencia. Un fragmento de dos vueltas (IGM 7780), otro, de una vuelta, y un fragmento chico, provienen de El Llano 1. El alcance estratigráfico de esta especie fue asignado al Caloviano inferior por Westermann et al. (1984). En este trabajo, por la asociación en la que fue colectado el material, se la asigna al Caloviano medio.

\section{El Caloviano de la región mixteca}

\subsection{Antecedentes}

En las investigaciones realizadas por Burckhardt (1927) en la región Mixteca de Oaxaca fueron establecidas las bases de la paleontología del Jurásico Medio del sur de México. En su monografía, la fauna de amonoideos está muy bien descrita y se señala que, conforme a la distribución del género Reineckeia en Europa, se debe admitir que la rica fauna de Reineckeia de El Consuelo, Oaxaca, indica con mucha probabilidad la existencia del Caloviano medio (Zona de Reineckeia ancep). Debe sin embargo subrayarse que Burckhardt no colectó la especie $R$. anceps, pero señaló la semejanza de la fauna oaxaqueña con la europea.

Erben (1956) cuando estudió las áreas de Tezoatlán, El Consuelo, Tlaxiaco y Mixtepec de la Mixteca de Oaxaca y estableció la nomenclatura formacional estratigráfica del Jurásico Medio de México, solamente proporcionó una lista de las especies calovianas que Burckhardt (1927) había presentado en su excelente monografía. Describió la Formación Yucuñuti, cuya localidad tipo se encuentra en Santa María Yucuñuti, Tezoatlán, Oaxaca, que aflora con un espesor de $50 \mathrm{~m}$ y consiste en lutitas amarillas, café o grises, e intercalaciones de areniscas conteniendo ammonites y bivalvos.

Westermann et al. (1984) denominaron asociación de Neuqueniceras a la fauna que estudiaron de la Formación Yucuñuti de Cualac, Guerrero; reportan que en la base de sus secciones se encontró Epistrenoceras aff. hystricoides (Rollier) junto con Choffatia suborion Burckhardt. La primera de estas especies se restringe en el Mediterráneo de Francia a la parte media del Batoniano superior. En el límite superior reportan por primera vez Clydoniceras inflatum n. sp, especie que se encuentra en Europa en la parte superior del Batoniano superior, Zona Discus. Le siguen los primeros representantes del género Reineckeia, que es el subgénero Rehmannia (y/o Tyranites), que en el Tethys se localiza en la Zona Macrocephalus. Esta secuencia, indican, se correlaciona con las estudiadas en el norte de Chile y el sur de Perú.

En su conclusión indican que la aparición de $R$. (Rehmannia / Tyrannites) en Guerrero, México ocurre antes que en Europa; ésto y su graduación hacia $N$. (F.) bodenbenderi sugiere que el origen de Reineckeiidae fue en el oriente del Pacífico y no en Europa.

En otro estudio de la misma área, Sandoval et al. (1990) complementaron el estudio señalado, y distinguen una zonificación para el Batoniano-Caloviano y lo subdividen en asociaciones de ammonites.

\subsection{Discusión}

La fauna descrita en este trabajo, correspondiente al Miembro Yucuñuti de la formación del mismo nombre en la Mixteca de Oaxaca, guarda cierta similitud con la descrita en los trabajos antes citados, pero también proporciona un mejor conocimiento y contribuye con nueva información bioestratigráfica para el Caloviano medio.

En el área de Mixtepec se estudiaron las localidades El Llano 1 y El Llano 2, separadas por una distancia de 500 $\mathrm{m}$, las cuales alcanzan la extensión vertical del Miembro Yucuñuti. Desde el punto de vista sedimentológico correlacionan muy bien si tomamos en cuenta dos puntos de referencia, uno es la discordancia que tienen aproximadamente a la mitad de la columna litológica y el otro los niveles de las mismas.

En esta misma área se reconoce claramente (Carrasco- 

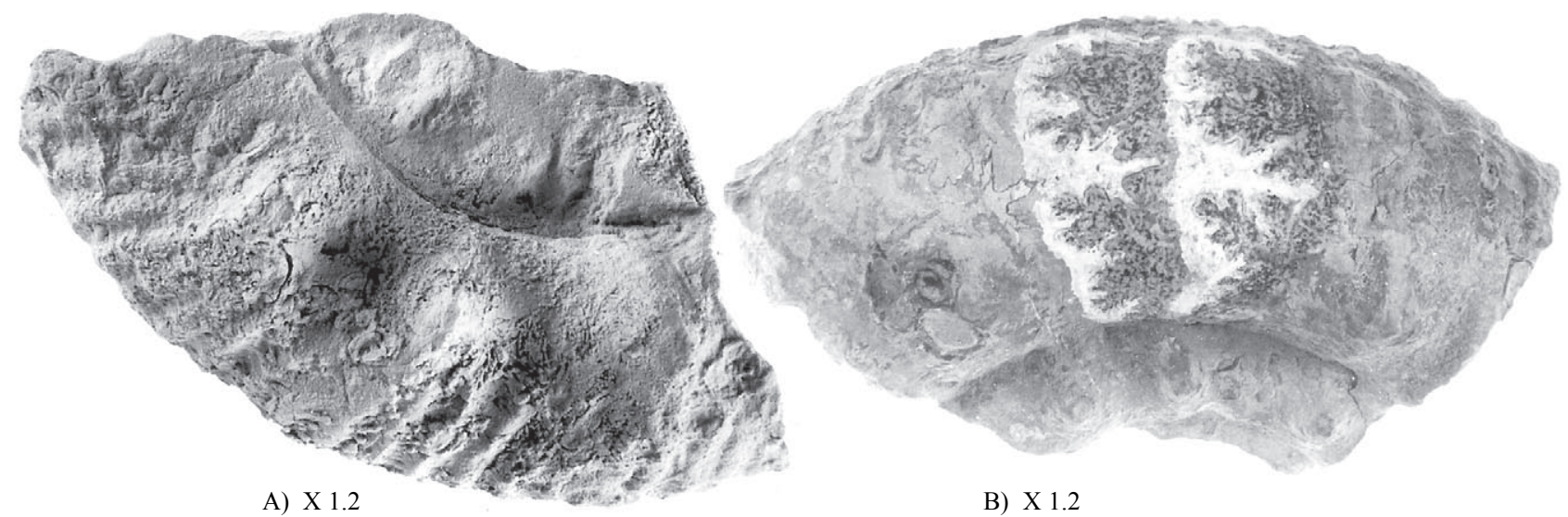

B) $\mathrm{X} 1.2$

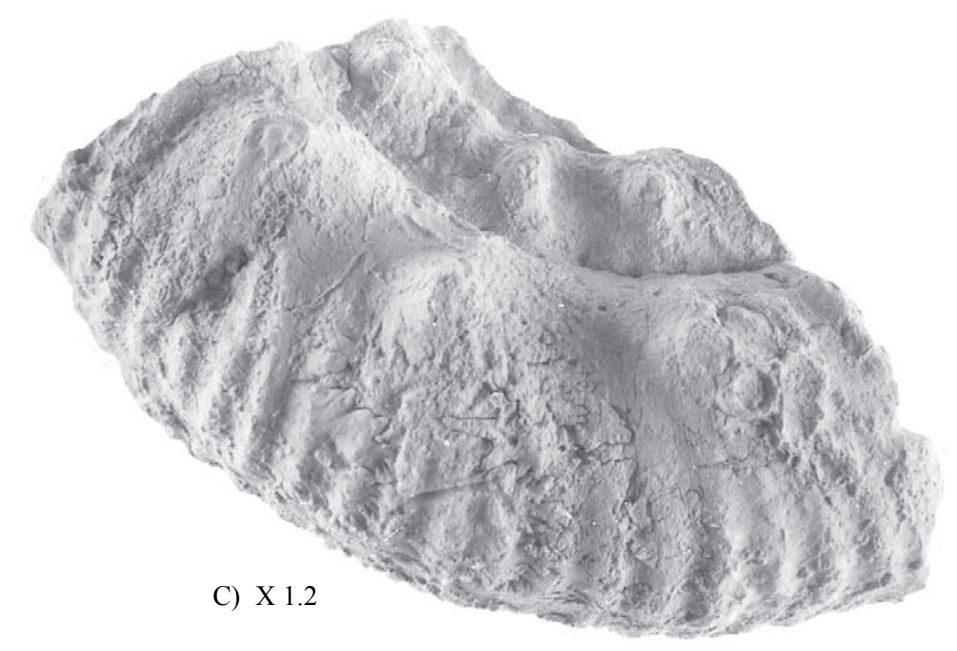

Figura 12. Reineckeia tuberosa Burckhardt, IGM-7780, localidad El Llano 1, en toda la extensión del Miembro Yucuñuti; A) vista lateral (X 1.2); B) vista latero-ventral (X 1.2), muestra detalle de la sutura; C) vista lateral (X 1.2).

Ramírez, 1999) una asociación de ReineckeiaNeuqueniceras (Nueva) (NACSN, 1983, p. 862). Para definir esta asociación se estudiaron las localidades mencionadas, donde se colectaron las especies $R$. tuberosa y $N$. inermis. La parte inferior de esta zona contiene las especies Rehmannia cf. grossouvrei (Petitclerc) y $R$. tuberosa (Burckhardt) en El Llano 1; Reineckeia (Reineckeia) anceps (Reinecke), Neuqueniceras (Frickites) cf. bodenbenderi (Tornquist) en El Llano 2. En la parte media de la sección El Llano 2 se encontró $R$. (R.) anceps (Reinecke) en asociación con $R$. tuberosa (Burckhardt), $N$. (F.) cf. bodenbenderi (Tornquist), ex situ Neuqueniceras cf. neogaeum (Burckhardt), Ch. cf. suborion (Burckhardt) y ex situ "Peltoceras" cricotum (Burckhardt). En la parte superior de las localidades El Llano 1 y El Llano 2 se colectó a $N$. inermis (Burckhardt) y ex situ Ch. praecursor (Mangold) respectivamente.

Rehmannia cf. grossouvrei se encuentra en Europa en las partes superior e inferior de las zonas estándar
Macrocephalus y Gracilis, respectivamente, base del Caloviano inferior (Sandoval et al., 1990). N. (F.) cf. bodenbenderi y $N$. cf. neogaeum fueron ubicados en el Caloviano inferior por Westermann et al. (1984), Riccardi et al.(1989) y Sandoval et al. (1990). R. (R.) anceps corresponde, en la zonación estándar europea, a la Zona Anceps, Subzona Stuebeli, base del Caloviano medio (Cariou y Krishna, 1988; Thierry et al., 1997).

La especie mediterránea $C$. praecursor se distribuye desde el Batoniano superior al Caloviano medio (Mangold, 1970). R. anceps se colectó en la localidad El Llano 2 en un intervalo de $38 \mathrm{~m}$, los cuales corresponden a la base del Caloviano medio. En la región de Cualac, Guerrero, Sandoval et al. (1990) colectaron previamente especímenes reportados en el Caloviano inferior, y de éstos, algunos (como ya se comentó) se volvieron a colectar en este trabajo. No obstante, los resultados obtenidos en esta investigación indican que las especies de la asociación ReineckeiaNeuqueniceras de la Mixteca de Oaxaca, tienen una 
distribución que alcanza hasta la base del Caloviano medio.

Como se comentó al inicio de este capítulo Burckhardt (1927, p. 98) indica que la distribución del género Reineckeia en Europa se puede correlacionar con la rica fauna de Reineckeia de El Consuelo en Oaxaca (a pocos kilómetros de S.J. Mixtepec), y señala que en esta área existe alta probabilidad de la existencia del Caloviano medio, es decir, la Zona de Reineckeia anceps.

En estudios efectuados por Westermann et al. (1984) se reporta que el Conjunto de Neuqueniceras se encuentra, con especies muy similares, en los Andes ArgentinoChilenos. Sandoval et al. (1990) señalan que la afinidad biogeográfica de esta fauna es primero con la Provincia Andina y segundo con la Provincia Mediterránea

En este trabajo se reconoce al género Choffatia, que es abundante en México, como cosmopolita. La especie $C$. (C.) praecursor es de origen Mediterráneo, en cambio la especie $C$. suborion se considera endémica del sur de México.

El género Neuqueniceras y la especie $N$. (F.) bodenbenderi son de origen andino, mientras que $N$. neogaeum y $N$. inermes son endémica de México. El género Rehmannia y R. grossouvrei son formas mediterráneas. Reineckeia y $R$. (R.) anceps son relativamente frecuentes en la Provincia Mediterránea, en tanto que $R$. tuberosa es endémica de México. El género Peltoceras es cosmopolita y $P$. cricotum es especie endémica de México.

En conclusión, los ammonites calovianos son en su mayoría afines a la Provincia Mediterránea, en segundo término guardan afinidad con la Provincia Andina, y existe una cantidad significativa de formas endémicas a México.

Los resultados obtenidos en los trabajos previos respecto a la distribución de las especies tienen cierta similitud con los aquí presentados, no obstante, las conclusiones que aquí se obtienen son diferentes, porque corresponden a secuencias más jóvenes que recibieron mayor influencia de la fauna proveniente del Tethys occidental.

Esta fauna existió hacia los finales del Dominio Pacífico Oriental, el que se desarrolló rápidamente en el Bajociano tardío y se dispersó más gradualmente en el Caloviano temprano. La sucesión faunística de la Mixteca indica una morfoinclinación filética, que lleva de Choffatia por la vía de Neuqueniceras y el grupo de N. (Frickites) bodembenderi, a Rehmannia y a la primera Reineckeia. Los Reineckeiidae se originaron en el Pacífico Oriental tropical en el Batoniano tardío expandiéndose de ahí hacia el Tethys occidental en el Caloviano temprano (Sandoval et al., 1990).

En el Caloviano, la familia Reineckeiidae comprende dos subfamilias que están separadas geográficamente, la del Pacífico, Neuqueniceratinae y la principalmente Tethysiana, Reineckeiinae; esta última representa probablemente una rama derivativa lateral del tronco Pacífico que se desarrolló ahí en el Batoniano tardío. A medida que Reineckeiidae penetró dentro del Tethys por la ruta de América Central, al inicio del Caloviano, dio nacimiento probablemente a la subfamilia Reineckeiinae.
Durante la mayor parte del Caloviano inferior, la distribución geográfica de la subfamilia esta restringida al Tethys occidental (Cariou y Krishna, 1988).

\section{Conclusiones}

En las localidades El Llano 1 y El Llano 2 de la Formación Yucuñuti se documenta por primera vez la presencia de $R$. anceps, fósil índice de la base del Caloviano medio, Zona Anceps, Subzona Stuebeli de la zonación de Europa ( Thierry, et al., 1997). También, como consecuencia de esto, se reporta una distribución más amplia para especies previamente estudiadas en Cualac, Guerrero por Westermann et al. (1984) y Sandoval et al. (1990).

La presencia de elementos de las faunas andinas y de las faunas mediterráneas, confirma que el Terreno Mixteco fue un pasaje al Pacífico Oriental de las faunas que vinieron del Mediterráneo circulando por el Corredor Hispánico.

El término Corredor Hispánico surgió posteriormente a las investigaciones realizadas por Hallam (1977) sobre la evidencia biogeográfica en los mares intermitentes del Jurásico. Y fue propuesto por Smith (1983) al estudiar la paleogeografía del Jurásico Temprano.

La fuerte afinidad de las asociaciones calovianas con la Provincia Mediterránea y la menor afinidad con las Provincias Andina va de acuerdo con la hipótesis paleogeográfica que considera al Terreno Mixteco colocado cerca de la entrada al Pacífico del Corredor Hispánico (Taylor et al., 1984; Westermann et al., 1984).

Las migraciones que se ven reflejadas en la fauna de ammonites estudiada de la Formación Yucuñuti, coinciden con elevaciones del nivel del mar en toda el área jurásica del Terreno Mixteco y, significativamente, también con los picos o elevaciones del nivel eustático de los océanos del mundo (Hallam, 1978), los que supuestamente removieron barreras en el pasaje marino-cratónico.

\section{Agradecimientos}

La presente investigación se realizó gracias al apoyo económico del CONACyT por medio de una beca-crédito y de la Geological Society of America (Grant 4071). Estos apoyos económicos permitieron al autor realizar los estudios y el trabajo de tesis doctoral con especialidad en Geología en la UNAM, México.

La Dra. Gloria Alencáster investigadora titular del Instituto de Geología de la UNAM, y profesor asesor del autor ayudó significativamente en la paleontología sistemática. La Dra. Blanca Buitrón investigadora titular del mismo instituto proporcionó una contribución invaluable en la realización del trabajo. El Dr. Alberto C. Riccardi de la Facultad de Ciencias Naturales y Museo de la Universidad Nacional de La Plata, La Plata, Argentina, experto en ammonites del Bajociano-Caloviano, accedió a leer y 
mejorar el manuscrito. Deseo agradecer especialmente al Dr. José Sandoval, especialista en ammonites del Jurásico Medio de la Universidad de Granada, España, quien contribuyó sustancialmente durante la elaboración del escrito final.

Por último deseo hacer patente mi agradecimiento a mi esposa Martha y a mi hijo Raúl Francisco quienes con su paciencia y apoyo se sacrificaron para que llegara terminar mi trabajo.

\section{Referencias bibliográficas}

Bayle, E., 1878, Fossiles principaux des terrains: Explication de la Carte Geologique de la France, 4, 158 p.

Bourquin, J., 1968, Les Reineckéidés: Annales Scientifiques de 1' Université de Besançon, Série 3, Géologie, 4, 169 p.

Burckhardt, C., 1927, Cefalópodos del Jurásico Medio de Oaxaca y Guerrero: Boletín del Instituto Geológico de México, 47, 108 p.

Cariou, E., 1980, L'étage Callovien dans le centre-ouest de la France: Poitiers, Francia, l'Université de Poitiers, tesis doctoral.

Cariou, E., Krishna, J., 1988, The Tethyan Reineckeiinae of Kachchh and Jaisalmer (west India); sistematic, biostratigraphic and biogeographic implications: Palaeontographica, Abteilung A, Palaeozoologie-Stratigraphie, 203, 149-170.

Carrasco-Ramírez, R.S., 1981, Geología Jurásica del área de Tlaxiaco, Mixteca Alta, Oaxaca: México, Universidad Nacional Autónoma de México, Facultad de Ciencias, tesis de maestría, 105 p.

Carrasco-Ramírez, R.S., 1999, Bioestratigrafia de amonitas (Cephalopoda-Ammonoidea) del Bajociano y Caloviano de una porción de la región Mixteca de Oaxaca: México, Universidad Nacional Autónoma de México, Instituto Geología, Posgrado en Ciencias de la Tierra, tesis doctoral, $91 \mathrm{p}$.

Erben, H.K., 1956, El Jurásico medio y el Calloviano de México, en $20^{\circ}$ Congreso Geológico Internacional: México, Universidad Nacional Autónoma de México, Instituto de Geología, 140 p.

Felix, J., 1891, Versteinerungen aus der Mexikanischen Jura und Kreide Formation: Palaeontographica, 37, 140-194.

Flores, T., 1909, Datos para la geología del estado de Oaxaca: Boletín de la Sociedad Geológica Mexicana, 5, 107-128.

Hallam, A., 1977, Biogeographic evidence bearing on the creation of Atlantic seaways in the Jurassic (with comments by G.E.G. Westermann, and reply), en West, R.W. (ed.), Paleontology and plate tectonics with reference for the history of the Atlantic ocean: Milwaukee Public Museum, Contributions in Biology and Geology, 2, 23-39.

Hallam, A., 1978, Eustatic cycles in the Jurassic: Palaeogeography, Palaeoclimatology, Palaeoecology, 23, 1-32.

Hyatt, A. 1900, Cephalopoda, en Zittel, K.V. (ed.), Textbook of Palaeontology: New York, MacMillan, 502-592.

Mangold, C., 1970, Les Perisphinctidae (Ammonitina) du Jura Meridional au Bathonienet au Callovien: Documents des Laboratoires de Geologie de la Faculte des Sciences de Lyon, 41, 246 p.

North American Commission on Stratigraphic Nomenclature (NACSN), 1983, North American Stratigraphic Code: American Association of Petroleum Geologists Bulletin, 67, 841-875.

Reinecke, M., 1818, Maris Protogaei Nautilos et Argonautas vulgo Cornua Ammonis in Agro Coburgico et vicino reperiondus: Cobourg, Ex officina et in commissis L.C.A. Ahlii, 90 p.
Riccardi, A.C., Westermann, G.E.G., Elmi, S., 1989, The Middle Jurassic Bathonian-Callovian ammonite Zones of the Argentine-Chilean Andes: Geobios, 22, 553-597.

Sandoval, G.J., Westermann, G.E.G., Marshall, M.C., 1990, Ammonite fauna stratigraphy and ecology of the Bathonian-Callovian (Jurassic) Tecocoyunca Group, south México: Palaeontographica, Abteilung A, Palaeozoologie-Stratigraphie, 210, 93-149.

Siemiradzki, J., 1898, Monographische Beschreibung der Ammonitengattung Perisphinctes: Palaeontographica (Stuttgart), $45,69-269$

Smith, P.L., 1983, The Pliensbachian Ammonite Dayiceras dayiceroides and Early Jurassic paleogeography: Canadian Journal of Earth Sciences, 20, 86-91.

Spath, I.E., 1930, The Jurassic ammonite faunas of the neighbourhood of Mombasa: University of Glasgow, Hunterian Museum, Monograph of the Geological Department, 4, 13-170.

Stehn, E., 1923, Beiträge zur Kenntnis des Bathonien und Callovien in Südamerika: Neues Jahrbuch der Mineralogie, Geologie und Palaontologie, 49, 52-158.

Steinmann, G., Doderlein, L.H.P., 1890, Elemente der Paläontologie: Leipzig, W. Engelmann, 848 p.

Taylor, D.G., Tipper, W.H., Westermann, G.E.G., Callomon, J.L., Hall, R., Smith, P.L., 1984, Jurassic ammonite biogeography of western North America: the tectonic implications, en Westermann, G.E.G. (ed.), Jurassic-Cretaceous biochronology and paleogeography of North America: Geological Association of Canada, Special Paper, 27, 121141.

Thierry, J., Cariou, E., Elmi, S., Mangold, C., Marchand, D., Rioult, M., 1997, Biozonations; Callovien, en Cariou, E., Hantzpergue, P. (eds.), Biostratigraphie du Jurassique ouest-européen et méditerranéen: zonations parallèles et distribution des invertebrés et microfossils; Groupe Francais d'étude de Jurassique, Callovien: Bulletin des Centre des Recherches Exploration-Production Elf-Aquitaine, 17, 63-78.

Waagen, W., 1869, Die formenreihe des Ammonites subradiatus: versuch einer palaontologischen monographie: GeognostischPaläontologische Beitrage, 2, 182-256.

Westermann, G.E.G., 1958, Ammoniten fauna und stratigraphie des Bathonien NW-Deutschlands: Hannover, Amt für Bodenforschung, $32,103 \mathrm{p}$

Westermann, G.E.G., 1984, Summary of symposium papers on the Jurassic-Cretaceous biochronology and paleogeography of North America, en Westermann, G.E.G. (ed.), Jurassic-Cretaceous biochronology and paleogeography of North America: Geological Association of Canada, Special Paper, 27, 307-315.

Westermann, G.E.G., Corona, R., Carrasco, R., 1984, The andean MidJurassic Neuqueniceras ammonite assemblage of Cualac, México, en Westermann, G.E.G. (ed.), Jurassic-Cretaceous biochronology and palaeogeography of North America: Geological Association of Canada, Special Paper, 27, 99-112.

Manuscrito recibido: Marzo 16, 2003

Manuscrito corregido recibido: Octubre 4, 2003

Manuscrito aceptado: Noviembre 10, 2003 